\section{PROMOCIÓN DE LA SALUD A TRAVÉS DE HERRAMIENTAS WEB 2.0. PROYECTO DE COLABORACIÓN ENTRE UNIVERSIDAD Y ENSEÑANZAS MEDIAS}

\author{
Patricia Florentín Dueñas \\ Departamento de Orientación. I.E.S. Bajo Aragón \\ pfd@unizar.es \\ Pedro José Satústegui Dordá \\ Departamento de Fisiatría y Enfermería. Universidad de Zaragoza \\ pjsd@unizar.es \\ Isabel Herrando Rodrigo \\ Departamento de Filología Inglesa y Alemana. Universidad \\ de Zaragoza \\ herrando@unizar.es \\ José Antonio Tobajas Asensio \\ Departamento de Fisiatría y Enfermería. Universidad de Zaragoza \\ jata@unizar.es
}

ABSTRACT: This project develops a highly innovative approach to a Health teaching and learning programme. Several didactic resources have been used and worked on thanks to the use of web 2.0 such and Blogger. The materials launched with these resources were created by Nursing undergraduates of the Escuela Universitaria de Ciencias de la Salud de la Universidad de Zaragoza (Spain), monitored by their teachers and used in an Aragonese high school -IES Bajo Aragón de Alcañiz (Teruel) - in order to improve the syllabus of their school tutorials -Plan de Acción Tutorial (PAT)-. Drawing on the results it could be stated that this project has empowered the health issues dealt with in the different years of Secondary Education in this high school.

KEY WORDS: PAT (Tutorial syllabus); health promotion; learning; free software as didactic resources.

\section{INTRODUCCIÓN}

Como parte del proceso de innovación que se está llevando a cabo a través del programa Escuela 2.0. (Orden de 15 de diciembre de 2009, para la aplicación del programa Escuela 2.0.) y ante la necesidad de educar a nuestros alumnos sobre temas de salud, desde el Departamento de Orientación

\section{PROMOTING HEALTH BY USING WEB 2.0: A COLLABORATIVE PROJECT BETWEEN TERTIARY AND SECONDARY EDUCATION}

RESUMEN: En este proyecto, se plantea un novedoso enfoque para la enseñanza-aprendizaje de temas de salud, mediante la utilización de recursos didácticos de la web 2.0. y software libre (Blogger). Para su desarrollo, se elaboraron materiales de trabajo por estudiantes de Enfermería de la Escuela Universitaria de Ciencias de la Salud de la Universidad de Zaragoza, con la finalidad de implementar un Programa de Promoción de Salud (PPS) dentro del Plan de Acción Tutorial (PAT) del Instituto de Educación Secundaria (IES) "Bajo Aragón" de Alcañiz (Teruel), promoviendo un empoderamiento en temas de salud en el alumnado de este centro.

PALABRAS CLAVE: Plan de Acción Tutorial; promoción de la salud; aprendizaje; software libre; recursos didácticos.

del IES "Bajo Aragón" de Alcañiz, se consideró la utilización de nuevas formas de exposición de contenidos como medio para complementar y dinamizar las sesiones de tutoría del alumnado de Educación Secundaria Obligatoria (ESO). Por otro lado, la Promoción de la Salud constituye un aspecto esencial dentro de las competencias que deben asumir los enfermeros que desarrollan su actividad en el campo de 
la Enfermería Familiar y Comunitaria (RD 450/2005, de 22 de abril, sobre especialidades de Enfermería).

Para dar respuesta a las necesidades de formación de ambos grupos de alumnos/as, se compilaron materiales educativos sobre temas de salud en la red utilizando la plataforma Blogger de uso libre, diseñando para ello un blog (disponible en http://saludenaragon.blogspot.com), para el que los estudiantes de la asignatura Enfermería Comunitaria I, que se cursa en segundo de Grado en Enfermería, en la Escuela Universitaria de Ciencias de la Salud de la Universidad de Zaragoza, elaboraron materiales que sirvieron como base a la implementación de un PPS que se desarrolló dentro del PAT del IES "Bajo Aragón" de Alcañiz.

De esta manera, se generó una intensa experiencia de aprendizaje para ambos grupos de estudiantes, que tuvo como elemento vertebrador un blog de elaboración ecléctica y que fue financiada con partidas presupuestarias procedentes del Gobierno de Aragón, de la Universidad de Zaragoza y del Fondo Social Europeo.

\section{Objetivos}

- Mejorar la calidad de la docencia en enseñanzas medias y universitarias a través de la combinación de herramientas de la WEB 2.0 y de la metodología del aprendizaje-servicio.

- Homogeneizar la planificación y los contenidos del PAT en el IES "Bajo Aragón" de Alcañiz.

- Promover la adquisición de competencias básicas sobre salud, en el alumnado de ESO del IES "Bajo Aragón" de Alcañiz.

- Desarrollar en el alumnado de segundo curso de Grado en Enfermería de la Universidad de Zaragoza, la capacidad para identificar necesidades, planificar y prestar servicios relacionados con la formación en temas de salud en un contexto real.

\section{Aspectos InNOVAdores del PROYeCto}

Nuestro proyecto incluyó una novedosa metodología de trabajo en las sesiones tutoriales de educación secunda- ria, que tuvo como base la utilización de materiales de la WEB 2.0. y la creación de un blog como sistema para organizarlos, lo que favoreció su uso por parte del profesorado y alumnado del instituto.

Los estudiantes de enseñanzas medias, en general, se sienten espacialmente atraídos por el uso y manejo de herramientas tecnológicas y muestran su preferencia por este tipo de soportes de información; La adecuación a las preferencias de una generación digital, favorece de manera decisiva el fomento de la lectura, que se realiza ahora sobre sustratos digitales e interactivos. Además, la utilización de diferentes plataformas y aplicaciones informáticas, contribuye a mejorar la competencia digital del alumnado participante, al someterlo a un constante ejercicio de adaptación y aprendizaje de los entornos utilizados.

Por otro lado, a través del trabajo propuesto a los estudiantes de Grado en Enfermería, en el que debian diseñar y elaborar materiales que iban a ser utilizados para la promoción de salud en el medio escolar, se contextualizó en un escenario real una actividad académica diseñada para conseguir el desarrollo de competencias profesionales en pregraduados, dotándola de sentido y finalidad, y transformándola en un elemento estimulador que promovió un aprendizaje más significativo.

\section{Desarrollo del proyecto}

El proyecto se desarrolló en 4 fases bien diferenciadas.

En la fase 0 se establecieron las bases del proyecto de colaboración entre el profesorado del Departamento de Orientación del IES y el profesorado del Departamento de Fisiatría y Enfermería de la Universidad Zaragoza, se definió la población diana a la que iría dirigido el PPS, se realizó el diagnóstico de necesidades educativas en materia de salud en estudiantes de tercero de ESO y se definieron conjuntamente las unidades temáticas que conformarian el PPS (Concepto de salud; Ejercicio físico y salud; Alimentación; Trastornos de la imagen corporal; Igualdad de género; Relaciones sexuales; Métodos anticonceptivos; Enfermedades de transmisión sexual; Accidentes de tráfico; Reanimación cardiopulmonar y Soporte Vital Básico; Consumo de Tabaco; Consumo de alcohol; Consumo de 
otras drogas). Por último, se diseñó un blog, que se utilizó para publicar los materiales elaborados.

En la fase 1 se establecieron los grupos de trabajo entre los alumnos/as universitarios/as y se realizaron tutorías de orientación dirigidas a facilitar la realización de materiales audiovisuales.

La fase 2 consistió en la selección y publicación de los materiales creados en el blog.

Durante la fase 3 se desarrollaron las unidades temáticas dentro del PAT del IES utilizando los materiales de trabajo publicados en el blog, recogiendo las aportaciones realizadas por los estudiantes de ESO y el feed-back recibido del alumnado universitario.

Por último, la fase 4 incluyó la evaluación y revisión del proyecto con el fin de conocer si el alumnado había adquirido las competencias básicas en materia de salud que se incluyen en el Currículo de tercero de ESO.

\section{Metodologías de aprendizaje utilizadas}

Dentro del Espacio Europeo de Enseñanza Superior (EEES) (Informe de ENQA sobre Criterios y Directrices para la Garantía de Calidad en el EEES, 2005), figura como objetivo estratégico el impulso en la implantación de nuevas metodologías de aprendizaje para conseguir una mayor calidad de la enseñanza. Por ello, y aprovechando el uso generalizado que nuestros alumnos hacen de Internet y de herramientas WEB 2.0. se implementó este proyecto con el que además se dio un importante servicio a la comunidad educativa.

Con el alumnado universitario, se utilizó un modelo formativo mixto de trabajo, en el que además de orientarle a la formación práctica, se le plantearon nuevas situaciones de aprendizaje en las que el profesorado actuaba como facilitador o guía (Torre y Gil, 2004), utilizando como base la metodología del aprendizaje-servicio (Puig, 2009).

En las aulas de enseñanza secundaria, se plantearon las sesiones tutoriales con los materiales colgados en el blog, siguiendo una metodología de trabajo tanto formal como informal, orientada hacia la adquisición de competencias, buscando la funcionalidad de los aprendizajes (Escamilla 2008), procurando fomentar la autonomía del alumnado y planteando situaciones que promovieran el debate y la reflexión. Al finalizar cada sesión, se administró al alumnado cuestionarios para valorar el grado de comprensión y adquisición de los contenidos desarrollados.

\section{Resultados}

Se utilizaron diferentes cuestionarios con la finalidad de evaluar la estructura, el proceso y los resultados obtenidos tras la ejecución del proyecto, siendo los que se presentan a continuación, los aspectos más relevantes.

\section{Resultados obtenidos en la evaluación realizada al alumnado universitario}

Un 78,79\% del alumnado consideró que elaborar en grupo un tema sobre promoción de salud dirigido a adolescentes utilizando las tecnologías de la información y la comunicación (TIC) le ayudó a tomar conciencia sobre la complejidad y las dificultades que plantea la educación en salud a la población, afirmando un $69,70 \%$ de los/as alumnos/as que esta actividad facilitó la adquisición de algunas de las competencias necesarias para trabajar en un futuro en el área de la Enfermería Comunitaria. Un 72,27\% de alumnado recomendaría al profesorado continuar con esta actividad el próximo curso y el 83,33\% consideró que la elección de los temas a desarrollar, la organización de los grupos y el tiempo para la preparación del trabajo fue adecuada o muy adecuada. Por último, el 69,7\% de los estudiantes valoraron con una nota superior a 7 la calidad de la actividad desarrollada, mientras que sólo un $24,24 \%$ la puntuó con notas que oscilaban entre 5 y 7 y un residual $6,06 \%$ lo hizo con una puntuación menor a 5 .

\section{Resultados obtenidos en la evaluación realizada al profesorado de ESO}

El profesorado encuestado se mostró conforme con la existencia de los blogs sobre temas de salud, considerando el 85,7\% que esta medida era "Muy adecuada". También fue unánime la valoración positiva de la calidad de los materiales, pues el $71,4 \%$ del profesorado los calificó como 
"Adecuados". Una gran mayoría, el 71,4\%, consideró que "No" sería necesario modificar los contenidos de los blogs ni los vídeos presentados, siendo la valoración numérica global que otorgaron al proyecto de 8,6 puntos (que se incrementó hasta 9 en la mediana), excelente valoración que también se aprecia al contemplar los valores máximo y mínimo, sobre todo este último, que fue de 7 puntos.

\section{Resultados obtenidos en la evaluación realizada al alumnado de ESO}

El alumnado de ESO estuvo formado en un 55,8\% por hombres y en un $44,2 \%$ por mujeres, y su perfil en cuanto a la edad, fue el de una persona de 15 años.

Una muy amplia mayoría de los/as alumnos/as (92,3\%) se mostraron conformes con el uso y la existencia de blogs sobre temas de salud y solo un 7,7\% los consideró inadecuados (Figura 1).

Consideras que el hecho de que exista una serie de blogs sobre temas de salud, para su utilización en la clase de tutoría es una medida...

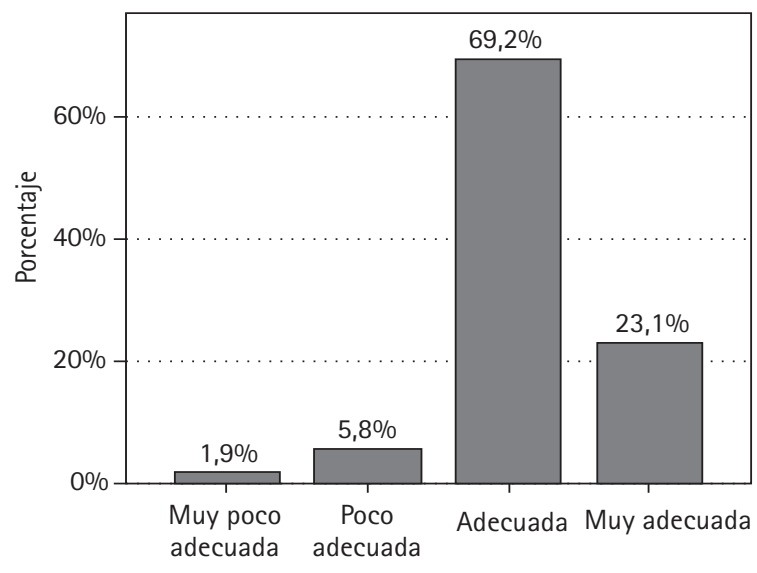

Figura 1. Opinión del alumnado de ESO sobre la utilidad del blog de salud en las sesiones de tutoría.

El contenido de los temas fue considerado por el 90,4\% de los/as alumnos/as como "Adecuado" o "Muy adecuado", y el 76,0\% consideró que "No" sería necesario modificar los contenidos de los blogs ni los vídeos presentados. En la valoración global del proyecto, se puede comprobar que la valoración media (y mediana) fue bastante alta, con
7,3 puntos (que se incrementó hasta 8 en la mediana) (Figura 2).

Valoración del proyecto

\begin{tabular}{|ll|c|}
\hline & & Estadísticos \\
\hline \multirow{2}{*}{$N$} & Válidos & 104 \\
\cline { 2 - 3 } & Perdidos & 0 \\
\hline Media & 7,3 \\
\hline Mediana & 8,0 \\
\hline Desv. típ. & 1,5 \\
\hline Mínimo & 3,0 \\
\hline Máximo & 10,0 \\
\hline Coef. Variación & $20,5 \%$ \\
\hline
\end{tabular}

Figura 2. Valoración global de los estudiantes de ESO del proyecto.

\section{Resultados obtenidos en la evaluación de las sesiones de tutoría}

Se consideró estudiar como variable de interés, el porcentaje de estudiantes que obtuvieron en las preguntas planteadas tras las sesiones de tutoría, porcentajes de aciertos iguales o mayores al 70\%. Tras el análisis estadístico, podemos afirmar que los resultados fueron excelentes, pues una amplia mayoría del alumnado obtuvo en los cuestionarios de los temas puntajes de aciertos iguales o superiores al 70\%. Es destacable el hecho de que el tema con mayor número de alumnos/as con porcentaje de aciertos por encima del 70\% fuera el de "Igualdad de género" (98,9\% del alumnado con al menos el $70 \%$ de respuestas correctas), mientras que el tema en el que menor número de estudiantes obtuvieron puntajes por encima del $70 \%$ de respuestas correctas fue el de "Enfermedades de transmisión sexual" (79,2\% de estudiantes con puntajes iguales o superiores al 70\%).

\section{SOSTENIBILIDAD Y TRANSFERENCIA DE LA ACTUACIÓN}

La propuesta presentada puede considerarse sostenible económicamente, especialmente si se cuenta con un profesorado (tanto de secundaria como universitario) altamente motivado, pues las horas que invierten los docentes en el trabajo son importantes. 
Por otro lado, la transferencia a otros escenarios docentes, donde se vean involucrados distintos actores es posible, pues la metodología del aprendizaje-servicio ha sido implementada en múltiples disciplinas (Puig, 2009) y su uso combinado con herramientas web 2.0, aumenta su aplicabilidad a distintos objetivos docentes.

\section{Conclusiones}

La utilización de una metodología de trabajo innovadora a través de la utilización de recursos de la WEB 2.0. ha supuesto una mejora considerable en la eficacia del PAT del IES Bajo Aragón de Alcañiz, favoreciendo la adquisición de las competencias relacionadas con la salud en el alumnado de ESO que cursa estudios en este centro.

La metodología del aprendizaje-servicio dentro del ámbito universitario, mejoró la calidad del proceso de enseñanza-aprendizaje, al permitir poner en práctica aspectos relacionados con el desarrollo de programas de salud en contextos reales, estimulando la proactividad en el alumnado y favoreciendo la adquisición de una serie de competencias profesionales en los pregraduados de Enfermería.

\section{BIBLIOGRAFÍA}

Escamilla, A. (2008): La competencias básicas: claves y propuesta para su desarrollo en centros. Barcelona, Ed. Graó.

European Association for Quality Assurance in Higher Education (2005): Criterios y Directrices para la Garantía de Calidad en el Espacio Europeo de Educación Superior, Helsinki. Consultado el 20 de mayo de 2011 en: http://www. eees.es/pdf/enqa_criteriosydirectrices_261005.pdf

Orden de 15 de diciembre de 2009, del vicepresidente del Gobierno, por la que se dispone la publicación del convenio de colaboración, entre el Ministerio de Educación y la Comunidad Autónoma de Aragón, para la aplicación del programa Escuela 2.0. BOA n. ${ }^{\circ}$, de 7 de enero de 2010.

Puig, J. M. (2009): Aprendizaje servicio (APS). Educación y compromiso cívico, Barcelona, Graó.

RD 450/2005, de 22 de abril, sobre especialidades de Enfermería, BOE n. ${ }^{\circ} 108$, de 6 de mayo de 2005.

Torre, J. C. y Gil, E. (2004): Hacia una enseñanza universitaria centrada en el aprendizaje, Alcobendas (Madrid), Gráficas Ormag. 